\title{
Long-term comparison of everolimus- vs. novolimus-eluting bioresorbable vascular scaffolds in real world patients
}

\author{
Beytullah Cakal, Sinem Cakal, Oguz Karaca, Mehmet Onur Omaygenc, Filiz Kizilirmak Yilmaz, \\ Haci Murat Gunes, Ozgur Ulas Ozcan, Arzu Yıldırım, Bilal Boztosun \\ Department of Cardiology, Istanbul Medipol University, Istanbul, Turkey
}

Adv Interv Cardiol 2020; 16, 4 (62): 391-398 DOI: https://doi.org/10.5114/aic.2020.101763

\begin{abstract}
A bstract
Introduction: Elevated risk of adverse events in comparison to metallic stents resulted in withdrawal of everolimus-eluting bioresorbable scaffolds (eBVS), known as the most intensively studied BVS. There is a paucity of data comparing the two different BVS.

Aim: To evaluate the long-term clinical outcomes of the novolimus-eluting bioresorbable vascular scaffold (nBVS) compared with eBVS.

Material and methods: Consecutive patients treated with nBVS or eBVS in our center were screened. The primary outcome was the 3-year rate of major adverse cardiovascular events (MACE), defined as the composite of cardiac death, target vessel myocardial infarction (TV-MI), and target-lesion revascularization (TLR).

Results: After matching, 98 patients treated with 135 eBVS were compared with 98 patients treated with 136 nBVS. Baseline characteristics, clinical presentation, and lesion characteristics were comparable in both groups. The 3-year MACE rate was higher in the eBVS group $(17.3 \%$ vs. $6.1 \%$; $p$ log-rank $=0.02)$. The occurrence of TLR $(16.3 \%$ vs. $5.1 \% ; p$ log-rank $=0.02)$ and TV-MI $(8.2 \%$ vs. $0 \% ; p$ log-rank $=0.004)$ was also higher in the eBVS group except for cardiac deaths $(1 \%$ vs. $2 \% ; p$ log-rank $=0.98$, eBVS vs. nBVS, respectively). Of note, definite device thrombosis rate was markedly increased in the eBVS group $(5.1 \%$ vs. $0 \%$; $p$ log-rank $=0.03)$.

Conclusions: The present study revealed that the 3 -year event risk was lower for nBVS compared to eBVS. More evidence is needed to evaluate long-term performance of novolimus-eluting biovascular platforms.
\end{abstract}

Key words: coronary artery disease, percutaneous coronary intervention, bioresorbable scaffolds.

S u m m a ry

The largest amount of evidence for bioresorbable vascular scaffold (BVS) use in clinical practice derives from Absorb trials and registries. Comparison of Absorb BVS with metallic stents resulted in increased rates of target lesion failure and device thrombosis in the Absorb BVS group. We investigated whether all BVS platforms created equal outcomes. At 3-year follow-up novolimus-eluting BVS (Desolve Elixir Medical Corp., Sunnyvale, CA) achieved better outcomes compared with Absorb BVS. The scaffold thrombosis rate is still high with Absorb BVS despite pre- and post- dilatation. No scaffold thrombosis was present with Desolve BVS.

\section{Introduction}

Bioresorbable vascular scaffolds (BVS) have represented a revolution in percutaneous coronary interventions $(\mathrm{PCl})$ as an alternative to drug-eluting stents (DES) with the goal of complete resorption to eliminate the risk of adverse events due to permanent presence of the metallic stents $[1,2]$.
The initial analysis of data with the first commercially available everolimus-eluting BVS (eBVS) (Absorb, Abbott Vascular, Santa Clara, CA, USA) in de novo simple lesions showed non-inferior outcomes to metallic DES in patients with stable coronary artery disease in shortterm follow-up [3]. However, long-term outcomes from randomized trials showed a significantly higher 3-year

Corresponding author:

Beytullah Cakal MD, Department of Cardiology, Istanbul Medipol University, Istanbul, Turkey, e-mail: bcakal@hotmail.com

Received: 6.06.2020, accepted: 22.08.2020. 
rate of scaffold thrombosis for eBVS [4]. Because of these concerns, on September 2017, the manufacturer stopped production of the first-generation Absorb BVS due to low sales. Due to the disappointing outcomes mentioned above, the implementation of BVS was given a class III indication in clinical practice outside of studies in the current European Society of Cardiology (ESC) guidelines [5].

Early positive results of eBVS encouraged many other companies to develop their own versions of BVS, one of which is the Novolimus-eluting BVS (nBVS) (DESolve, Elixir Medical Corporation, Sunnyvale, California, USA), which received the CE mark in May 2014. Sufficient data evaluating either direct or indirect comparison of these two different BVS are presently not available in everyday clinical practice. Therefore, the purpose of this study was to compare clinical outcomes of the two BVS in a real-world population.

\section{Aim}

The purpose of the present study based on single-center registry was to compare the long-term outcomes of real-world patients treated with nBVS or eBVS.

\section{Material and methods}

\section{Study design and population}

This was an observational, retrospective, single-center study of consecutive patients performed in a high-volume, tertiary $\mathrm{PCl}$ center, Istanbul, Turkey with an annual $\mathrm{PCl}$ volume of approximately 3000 procedures. The study population consisted of all patients who underwent PCI with at least one eBVS or nBVS implantation for coronary heart disease between March 2013 and February 2018. The nBVS has been available for implantation since May 2015. The decision to treat the lesion with either eBVS or nBVS was left to the operator's choice. All clinical data at follow-up were collected from hospital visits or telephone consultations for all patients. The study was approved by the ethics board of Istanbul Medipol University, Turkey.

Eligible patients were those with reference vessel diameter (RVD) $\geq 2.50 \mathrm{~mm}$, stentable lesions, stable coronary artery disease, or acute coronary syndrome. All treated lesions consisted of stenosis $>50 \%$ in native coronary arteries. Major exclusion criteria were defined as left main coronary artery lesions, saphenous vein graft lesions, presence of lesions requiring stents greater than $4.0 \mathrm{~mm}$ and less than $2.5 \mathrm{~mm}$, and patients implanted with both nBVS and eBVS. No restrictions were applied for the number of lesions and vessels treated, lesion length or the number of implanted stents.

\section{Study devices}

The eBVS consists of an amorphous matrix of polyL-lactic acid (PLLA), coated with a layer of a $1: 1$ mixture of poly-D-L-lactic acid and $100 \mathrm{\mu g} / \mathrm{cm}^{2}$ anti-inflammatory everolimus. eBVS is manufactured using extrusion and laser machining techniques, featuring $157 \mu \mathrm{m}$ strut thickness and comprising in-phase zig-zag hoops linked with a bridge design. The full dissolution process requires 2-3 years to complete [1].

The nBVS is made from a PLLA-derived polymer backbone similar to that of the eBVS and has a strut thickness of $150 \mu \mathrm{m}$ with an average vessel area coverage of $30 \%$ for a $3.0 \mathrm{~mm}$ vessel stent. More than $85 \%$ of Novolimus at a $3 \mu \mathrm{g} / \mathrm{mm}$ dose is released over 4 weeks with $>95 \%$ loss of molecular mass in the first year. The two most remarkable features of nBVS are intrinsic unique self-correcting deployment properties to improve minor strut malapposition and relatively high elasticity with fracture resistance of the scaffold (e.g. $3.0 \mathrm{~mm}$ nBVS up to $20 \mathrm{~atm}$ with a $5 \mathrm{~mm}$ balloon) [6].

\section{Procedure}

The choice of BVS was left to the operator's discretion and the decision for BVS implantation according the principles of PSP (predilation, appropriate sizing, and post-dilation to high pressures) technique was not mandatory but highly recommended. Generally, pre-dilation was performed with compliant or non-compliant balloons, while for more calcified lesions a scoreflex balloon (Orbus Neich, Hong Kong) Angio Sculpt Scoring Balloon (AngioScore, Inc) was preferred. Post-dilatation was performed with non-compliant balloons with the same size of BVS or $0.25-0.5 \mathrm{~mm}$ larger.

Quantitative coronary angiography (QCA) was performed offline using standard techniques with automated edge detection algorithms (CAAS 5.7.1, Pie Medical Imaging, Maastricht, The Netherlands) in the hospital's angiographic analysis center.

\section{Postprocedural management and follow-up}

Patients were treated with clopidogrel (600 mg) or prasugrel $(60 \mathrm{mg})$ or ticagrelor $(180 \mathrm{mg}$ ) loading doses before $\mathrm{PCl}$ followed with daily doses of clopidogrel $(75 \mathrm{mg})$ or prasugrel $(10 \mathrm{mg})$ or ticagrelor $(180 \mathrm{mg})$ for 12 months with daily oral aspirin of $100 \mathrm{mg}$. Clinical follow-up was achieved by medical visits, when patients could not attend visits, they were called and questioned for symptoms.

\section{Outcomes and definitions}

The primary outcomes of the study were the patient-oriented composite events (MACE), which included the composite of cardiac death, target vessel myocardial infarction (TV-MI), and clinically driven target lesion revascularization (TLR). Target vessel revascularization (TVR) was defined as repeat PCI or coronary artery bypass graft $(C A B G)$ in the target vessel. Deaths were considered cardiac unless a non-cardiac cause was identified. All components of the composite endpoints, and definite thrombosis, were classified according to the Academic Research Consortium [7]. 
Table I. Baseline clinical characteristics

\begin{tabular}{|c|c|c|c|c|c|c|}
\hline \multirow[t]{2}{*}{ Parameter } & \multicolumn{3}{|c|}{ Before propensity matching } & \multicolumn{3}{|c|}{ After propensity matching } \\
\hline & nBVS $(n=140)$ & eBVS $(n=98)$ & $P$-value & nBVS $(n=98)$ & eBVS $(n=98)$ & $P$-value \\
\hline Age [years] & $57 \pm 9.7$ & $60 \pm 11.5$ & 0.03 & $58 \pm 10$ & $60 \pm 11.5$ & 0.17 \\
\hline Male & $119(85 \%)$ & $79(81 \%)$ & 0.37 & $81(83 \%)$ & $79(81 \%)$ & 0.71 \\
\hline Smoking history & $72(51 \%)$ & $40(41 \%)$ & 0.11 & $44(45 \%)$ & $40(41 \%)$ & 0.56 \\
\hline Hypertension & $87(62 \%)$ & $62(63 \%)$ & 0.86 & $64(65 \%)$ & $62(63 \%)$ & 0.77 \\
\hline Hyperlipidemia & $85(61 \%)$ & $64(65 \%)$ & 0.47 & $63(64 \%)$ & $64(65 \%)$ & 0.88 \\
\hline Diabetes & $50(36 \%)$ & $37(38 \%)$ & 0.75 & $33(34 \%)$ & 37 (38\%) & 0.55 \\
\hline Previous MI & $35(25 \%)$ & $22(22 \%)$ & 0.65 & $28(28 \%)$ & $22(22 \%)$ & 0.33 \\
\hline Previous CABG & $5(3.6 \%)$ & $4(7 \%)$ & 0.22 & $4(4 \%)$ & $4(7 \%)$ & 0.35 \\
\hline Family history of CAD & 47 (34\%) & $36(37 \%)$ & 0.61 & $28(29 \%)$ & $36(37 \%)$ & 0.22 \\
\hline New P2Y12 inhibitor & $86(61 \%)$ & $53(54 \%)$ & 0.26 & $59(60 \%)$ & $55(54 \%)$ & 0.39 \\
\hline Left ventricle ejection fraction (\%) & $56 \pm 7$ & $56 \pm 9.5$ & 0.97 & $56 \pm 7$ & $56 \pm 9.5$ & 0.9 \\
\hline Heart failure & $5(3.6 \%)$ & $5(5 \%)$ & 0.56 & $4(4 \%)$ & $5(5 \%)$ & 0.73 \\
\hline \multicolumn{7}{|l|}{ Clinical presentation table: } \\
\hline Angina & $116(83 \%)$ & $1(83 \%)$ & 0.97 & $78(83 \%)$ & $81(80 \%)$ & 0.58 \\
\hline Acute coronary syndrome & $24(17 \%)$ & $17(17 \%)$ & & $20(17 \%)$ & $17(20 \%)$ & \\
\hline SYNTAX Score & $19.5 \pm 7$ & $18.6 \pm 6.9$ & 0.3 & $19.1 \pm 6.7$ & $18.6 \pm 6.9$ & 0.55 \\
\hline Total scaffold length [mmª & $28(28-56)$ & $28(23-36)$ & $0.15^{\mathrm{a}}$ & $28(28-56)$ & $28(23-36)$ & $0.26^{b}$ \\
\hline Procedural success, $n$ (\%) & 139 (99) & $96(98)$ & 0.57 & $97(99)$ & $96(98)$ & 1 \\
\hline
\end{tabular}

a Median (interquartile range); ${ }^{b}$ Mann-Whitney test. MI- myocardial infarction, CABG - coronary artery bypass grafting, CAD - coronary artery disease.

\section{Statistical analysis}

Values are presented as mean \pm standard deviation (SD) or median (interquartile range (IQR)) for continuous variable or as counts and percentages for categorical variables. The Mann-Whitney $U$ test or the independent sample $t$ test was used for comparison of continuous data and Fisher's exact test or the $\chi^{2}$ test was used to compare categorical data. Because of the non-randomized nature of the study, propensity score analysis (Fuzzy Python-based extension for SPSS) was used to adjust for differences in patients' baseline characteristics including age, sex, diabetes, smoking, hypertension, hypercholesterolaemia, previous $\mathrm{MI}$, acute coronary syndrome, family history of coronary artery disease, left ventricular ejection fraction, previous $\mathrm{PCI}$ history, previous CABG history, SYNTAX score, and total stent length. Time-to-event variables are presented as Kaplan-Meier curves. $P$-values of $<0.05$ were considered significant. Analyses were carried out using SPSS for Windows, version 25.0 (IBM Corp., Armonk, N.Y., USA).

\section{Results}

\section{Baseline characteristics}

Baseline characteristics of the overall and propensity matched cohort are presented in Table I. A total of 140 patients with nBVS $(n=202)$ and 98 patients with eBVS $(n=135)$ were found to be eligible for matching (Figure 1). Of these, 98 patients treated with 135 eBVS were matched 1 : 1 with 98 patients treated with 136 nBVS.

The proportions of patients with stable angina pectoris ( $83 \%$ vs. $80 \%$ ), acute coronary syndrome (17\% vs. $20 \%)$ were comparable in the two matched groups. The SYNTAX score (19.1 \pm 6.7 vs. $18.6 \pm 6.9 ; p=0.55)$ and total scaffold length (median: $28 \mathrm{~mm}$, IQR: 28-56; median: 28, IQR 23-36, $p=0.26$ ) did not differ between nBVS and eBVS groups, respectively. There were no differences in terms of procedural success between the two groups (98\% vs. $99 \%$ in eBVS vs. nBVS groups, respectively). In the nBVS group, one patient suffered side branch occlusion, whereas in eBVS there was one scaffold rupture during postdilatation and the second patient had TIMI 1 flow after overlapping of two scaffolds.

\section{Procedural characteristics and quantitative coronary angiography}

Angiographic and procedural characteristics of the overall cohort are summarized in Table II and the matched group is presented in Table III. Longer scaffolds were opted in the nBVS group (median: $28 \mathrm{~mm}$, (IQR) 28-28 for nBVS vs. median $28 \mathrm{~mm}$ (IQR) 18-28 mm for eBVS; $p=0.002$ ). Overlapping of two BVS was performed in 32 (23.7\%) vs. $32(23.5 \%)$ lesions in eBVS and nBVS groups respec- 


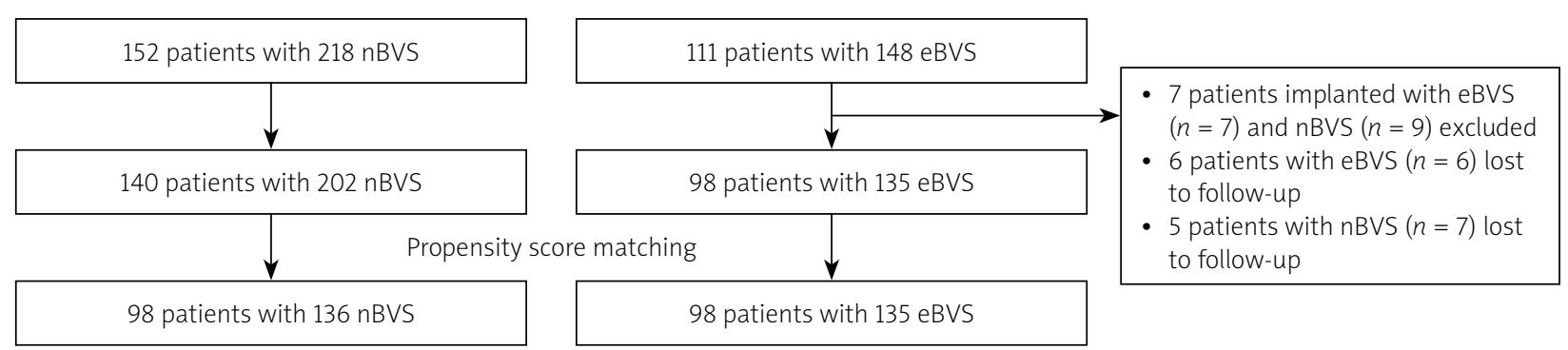

Figure 1. Study flowchart

Table II. Lesion and procedural characteristics in overall cohort

\begin{tabular}{|c|c|c|c|}
\hline Parameter & nBVS $(n=202)$ & eBVS $(n=135)$ & $P$-value \\
\hline Vessels treated, $n(\%)$ : & & 0.73 & \\
\hline$L A D$ & $123(61)$ & $70(52)$ & 0.1 \\
\hline$\underline{L C X}$ & $29(14)$ & $22(16)$ & 0.62 \\
\hline RCA & $50(25)$ & $43(32)$ & 0.15 \\
\hline \multicolumn{4}{|l|}{ Access route, $n(\%)$ : } \\
\hline Femoral & $51(37)$ & $82(84)$ & $<0.001$ \\
\hline Radial & $89(63)$ & $16(16)$ & \\
\hline \multicolumn{4}{|l|}{ QCA analysis: } \\
\hline Stenosis percentage $[\mathrm{mm}]$ & 72.713 & $67.5 \pm 114.9$ & 0.001 \\
\hline $\mathrm{RVD}[\mathrm{mm}]$ & $3.11 \pm 0.46$ & $3.16 \pm 0.43$ & 0.25 \\
\hline Pre MLD [mm] & $0.84 \pm 0.42$ & $1.02 \pm 0.49$ & $<0.001$ \\
\hline Post MLD [mm] & $2.79 \pm 0.38$ & $2.62 \pm 0.46$ & $<0.001$ \\
\hline Acute gain [mm] & $1.95 \pm 0.52$ & $1.59 \pm 0.6$ & $<0.001$ \\
\hline Predilatation balloon size [mm] & $2.87 \pm 0.42$ & $2.88 \pm 0.45$ & 0.72 \\
\hline Postdilatation balloon size [mm] & $3.29 \pm 0.43$ & $3.27 \pm 0.47$ & 0.66 \\
\hline Predilatation, $n(\%)$ : & $100 \%$ & $100 \%$ & \\
\hline Scoring balloon & $20 \%$ & $55 \%$ & $<0.001$ \\
\hline Cutting balloon & $1 \%$ & $0 \%$ & 1 \\
\hline Scaffold diameter [mm] & $3.1 \pm 0.44$ & $3 \pm 0.41$ & 0.37 \\
\hline Scaffold length $[\mathrm{mm}]^{a}$ & $28(28-28)$ & $28(18-28)$ & $0.05^{b}$ \\
\hline Number of BVS per lesion & $1.21 \pm 0.47$ & $1.15 \pm 0.36$ & 0.24 \\
\hline Scaffold length per lesion [mm] & $31 \pm 13$ & $28 \pm 10.4$ & 0.03 \\
\hline Postdilatation, $n(\%)$ & $199(99)$ & $128(95)$ & 0.1 \\
\hline \multicolumn{4}{|l|}{ Type of lesion, $n(\%)$ : } \\
\hline A/B1 & $135(66)$ & $80(59)$ & 0.16 \\
\hline $\mathrm{B} 2 / \mathrm{C}$ & $67(34)$ & $55(41)$ & \\
\hline Bifurcated & 39 (19.3) & $33(24)$ & 0.26 \\
\hline Calcified & $55(28)$ & $40(30)$ & 0.6 \\
\hline СTO & $7(3.5)$ & $7(5)$ & 0.44 \\
\hline Overlap & $103(51)$ & $62(46)$ & 0.36 \\
\hline In-stent restenosis & $2(1)$ & $6(4.4)$ & 0.06 \\
\hline
\end{tabular}

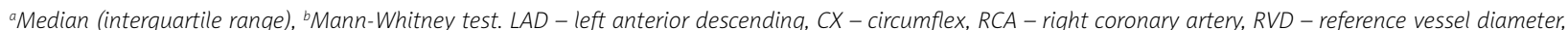
$M L D$ - minimum lumen diameter, BVS - bioresorbable vascular scaffold, CTO - chronic total occlusion.

tively, whereas overlapping with DES was performed in $30(22.2 \%)$ vs. $34(25 \%)(p=0.67)$ in eBVS and nBVS groups, respectively. The two groups were well balanced according to the lesion complexity including lesion types, bifurcation, CTO, calcification and in-stent restenosis rate.
Predilation was performed in every case, but postdilatation was more often used in the nBVS group ( $p=0.04)$. The acute gain value was significantly higher in nBVS compared to eBVS $(1.95 \pm 0.55$ vs. $1.59 \pm 0.6 ; p<0.001)$. The use of newer P2Y12 inhibitors was also similar $(p=0.39)$. 
Table III. Adjusted lesion and procedural characteristics (after propensity score matching)

\begin{tabular}{|c|c|c|c|}
\hline Parameter & nBVS $(n=136)$ & eBVS $(n=135)$ & $P$-value \\
\hline Vessels treated, $n(\%)$ : & & 0.73 & \\
\hline$\underline{L A D}$ & $83(61)$ & $70(52)$ & 0.13 \\
\hline LCX & $20(15)$ & $22(16)$ & 0.72 \\
\hline RCA & $33(24)$ & $43(32)$ & 0.16 \\
\hline \multicolumn{4}{|l|}{ Access route, $n(\%)$ : } \\
\hline Femoral & $35(36)$ & $82(84)$ & $<0.001$ \\
\hline Radial & $63(64)$ & $16(16)$ & \\
\hline \multicolumn{4}{|l|}{ QCA analysis: } \\
\hline Stenosis percentage $[\mathrm{mm}]$ & $73 \pm 13.3$ & $67.5 \pm 14.4$ & 0.001 \\
\hline$\underline{\mathrm{RVD}}[\mathrm{mm}]$ & $3.13 \pm 0.46$ & $3.16 \pm 0.43$ & 0.45 \\
\hline Pre MLD [mm] & $0.83 \pm 0.43$ & $1.03 \pm 0.48$ & $<0.001$ \\
\hline Post MLD [mm] & $2.78 \pm 0.39$ & $2.62 \pm 0.46$ & 0.002 \\
\hline Acute gain $[\mathrm{mm}]$ & $1.95 \pm 0.55$ & $1.59 \pm 0.6$ & $<0.001$ \\
\hline Predilatation balloon size [mm] & $2.87 \pm 0.4$ & $2.88 \pm 0.4$ & 0.90 \\
\hline Postdilatation balloon size [mm] & $3.29 \pm 0.42$ & $3.27 \pm 0.47$ & 0.62 \\
\hline \multicolumn{4}{|l|}{ Procedural data: } \\
\hline Predilatation (\%) & $100 \%$ & $100 \%$ & \\
\hline Scoring balloon & $22(16 \%)$ & $74(55 \%)$ & $<0.001$ \\
\hline Cutting balloon & $1(0.7 \%)$ & $0 \%$ & 1 \\
\hline$\underline{\text { Stent/scaffold diameter [mm] }}$ & $3.1 \pm 0.44$ & $3.1 \pm 0.41$ & 0.38 \\
\hline Scaffold length $[\mathrm{mm}]^{\mathrm{a}}$ & $28(28-28)$ & $28(18-28)$ & $0.002^{b}$ \\
\hline Number of BRS per lesion & $1.18 \pm 0.54$ & $1.15 \pm 0.36$ & 0.58 \\
\hline Scaffold length per lesion [mm] & $30 \pm 12.2$ & $28 \pm 10.5$ & 0.12 \\
\hline Postdilatation, $n(\%)$ & $135(99)$ & $128(95)$ & 0.04 \\
\hline \multicolumn{4}{|l|}{ Type of lesion, $n(\%)$ : } \\
\hline $\mathrm{A} / \mathrm{B} 1$ & $90(66)$ & $80(59)$ & 0.24 \\
\hline $\mathrm{B} 2 / \mathrm{C}$ & $46(34)$ & $55(41)$ & 0.24 \\
\hline Bifurcated & $28(21)$ & $33(24)$ & 0.44 \\
\hline Calcified & $30(22)$ & $40(30)$ & 0.17 \\
\hline CTO & $4(3)$ & $7(5)$ & 0.35 \\
\hline Overlap & $66(49)$ & $62(46)$ & 0.67 \\
\hline In-stent restenosis & $2(1.5)$ & $6(4.4)$ & 0.17 \\
\hline
\end{tabular}

a Median (interquartile range), "'Mann Whitney test. $L A D$ - left anterior descending, CX - circumflex, RCA - right coronary artery, RVD - reference vessel diameter, $M L D$ - minimum lumen diameter, BVS - biovascular scaffold, CTO - chronic total occlusion.

\section{Clinical outcomes in the propensity matched} cohort

Clinical outcomes in the matched population are presented in Table IV. Mean follow-up time for patients implanted with eBVS was $52.9 \pm 13.9$ months and $36.4 \pm 8$ months for nBVS treated patients $(p<0.001)$. The 3-year follow-up rate was $90 \%$ in eBVS and $82 \%$ in the nBVS groups. In the propensity matched cohort, at 3-year follow-up, theestimated cumulativeMACEincidence (Figure2) and its singular components were significantly higher in the eBVS group (MACE: 17.3 vs. $6.1 \% ; p_{\text {log-rank }}=0.02$, TLR: $16.3 \%$ vs. $5.1 ; p_{\text {log-rank }}=0.02$, TV-MI: $0 \%$ Vs. $8.2 \% ; p_{\text {log-rank }}$ $=0.004)$ except for cardiac deaths ( $1 \%$ vs. $2 \%$; $p_{\text {log-rank }}=$ 0.55 , for nBVS vs. eBVS, respectively). Scaffold thrombosis involving both early and very late events was detected in the eBVS group (5.1\%), but no case of definite scaffold thrombosis was present in the nBVS group ( $p$ log-rank = 0.03). The main cause of one non-cardiac death in the nBVS group was pancreatitis, occurring 23 months later. Moreover, the same patient was dialysis-dependent due to contrast nephropathy after nBVS implantation, whereas non-cardiac deaths were linked to gastric and prostate cancers at 23 and 24 months after eBVS implantation.

\section{Discussion}

The present study is the first to report mid-term comparative analysis of nBVS and eBVS outcomes based on propensity score matching. We found that 3-year incidence of MACE mainly driven by higher TLR and MI as well as early and late scaffold thrombosis were higher for eBVS compared to nBVS. Considering that BVS use leads to increased adverse events predominantly driven 
Table IV. Clinical outcomes at 1-year and 3-year follow-up

\begin{tabular}{lccc} 
Parameter & $\begin{array}{c}n \text { nVS } \\
(n=98)\end{array}$ & $\begin{array}{c}\text { eBVS } \\
(n=98)\end{array}$ & $\begin{array}{c}\text { P-value } \\
\text { (log-rank) }\end{array}$ \\
\hline Clinical outcome at 1 year: & & \\
\hline All-cause death & $1(1 \%)$ & $1(1 \%)$ & 0.99 \\
\hline Cardiac death & $1(1 \%)$ & $1(1 \%)$ & 0.99 \\
\hline TV-Ml & $0(0 \%)$ & $6(6.1 \%)$ & 0.01 \\
\hline Definite ST & $0(0 \%)$ & $3(3.1 \%)$ & 0.08 \\
\hline TVR & $2(2 \%)$ & $8(8.2 \%)$ & 0.06 \\
\hline TLR & $1(1 \%)$ & $8(8.2 \%)$ & 0.02 \\
\hline MACE & $2(2 \%)$ & $10(10.2 \%)$ & 0.02 \\
\hline Clinical outcome at 3 year: & & \\
\hline All-cause death & $2(2 \%)$ & $4(4.1 \%)$ & 0.43 \\
\hline Cardiac death & $1(1 \%)$ & $2(2 \%)$ & 0.98 \\
\hline TV-MI & 0 & $8(8.2 \%)$ & 0.004 \\
\hline Definite ST & 0 & $5(5.1 \%)$ & 0.03 \\
\hline TVR & $6(6.1 \%)$ & $16(16.3 \%)$ & 0.03 \\
\hline TLR & $5(5.1 \%)$ & $16(16.3 \%)$ & 0.02 \\
\hline MACE & $6(6.1 \%)$ & $17(17.3 \%)$ & 0.02 \\
\hline
\end{tabular}

MACE - major adverse cardiac event, TV-MI - target vessel myocardial infarction, $T L R$-target lesion revascularization, TVR-target vessel revascularization, ST - scaffold thrombosis.

by the results of eBVS trials, these concerns might not be attributed to nBVS.

After initial enthusiasm in relation to BVS, worse clinical outcomes in terms of TLR and scaffold thrombosis of eBVS, known as the most comprehensively studied BVS, have hampered the clinical use of other BVS [8]. A meta-analysis of the 4 randomized eBVS trials assigning patients to eBVS $(n=2164)$ or DES $(n=1225)$ resulted in higher 3-year rates of target lesion failure in the Absorb BVS group (11.7\% vs. 8.1\%; $p=0.006)$ [4]. Therefore, the use of BVS has been restricted to clinical studies [9].

Preliminary data from the 5-year follow-up for the DESolve $\mathrm{Nx}$ yielded no definite scaffold thrombosis, 4 cardiac deaths $(2.5 \%)$, and 5 clinically driven TLRs (4.1\%). The MACE rate was $7.4 \%$ at 2 years [10]. Relatively low-risk lesion subsets (mean lesion length 11.2 $\pm 3.8 \mathrm{~mm}$, Class B2/C lesion rate was $34 \%$ ) were present in that study in contrast to the current study. Multicenter, post-marketing evaluation of nBVS (100 patients treated with 109 lesions) demonstrated no cardiac deaths, one definite scaffold thrombosis (1\%), one MI (1\%) and three device-oriented composite endpoint (DOCEs) (3\%) at 1 year follow-up [11].

Presently, there are limited data on nBVS in comparison to the eBVS. Reports regarding optical coherence tomography (OCT) and bench comparison of nBVS with eBVS showed that eBVS has fewer peaks per hoop (6 vs. 9 ), smaller vessel coverage ("footprint"; $27 \%$ vs. $30 \%$ ), and higher radial strength than the nBVS (1.3 atm vs. 1.1 atm) while nBVS provided smaller residual area stenosis (20.1\% vs. $14.9 \% ; p<0.01)$ and larger maximum $(3.2 \mathrm{~mm}$ vs. $3.5 \mathrm{~mm} ; p=0.01)$ and minimum ( $2.7 \mathrm{~mm}$ vs. $2.9 \mathrm{~mm}$; $p<0.01)$ scaffold diameters with no strut fracture, suggesting better expansion of the nBVS $[12,13]$. Another bench test comparing Magmaris (Biotronik AG, Bülach, Switzerland), Absorb and DESolve BVS showed that Absorb and Magmaris have larger recoil rates and only DESolve did not fracture during side branch dilatation and mini-kissing balloon postdilatation [14]. In our study, QCA analysis showed larger post-procedure minimum lumen diameter (MLD) in the nBVS group despite similar baseline angiographic RVD measured in both groups. Nevertheless, our study does not address the influence of mechanical properties of BVS on clinical outcomes due to the absence of imaging modalities and randomization.

Although no randomized controlled trials or real life studies have been carried out for comparison of nBVS to eBVS, a single center, retrospective comparative analysis using propensity-score matching between eBVS and nBVS (approximately $85 \%$ post-dilation rate in both groups with shorter lesion length of $13 \mathrm{~mm}$ ) showed similar 1-year outcomes in terms of target lesion failure, cardiac death and scaffold thrombosis (1-year target lesion failure 4.7\% vs. $4.5 \% ; p=0.85$, respectively), contrary to positive findings for nBVS in the current study [15].

Another noteworthy finding of the current study was absence of scaffold thrombosis in the nBVS group. Scaffold thrombosis has been considered as a consequence of errors during implantation such as inadequate vessel sizing and malapposition [16]. Very late thrombosis and/or restenosis is triggered by scaffold discontinuity of uncovered scaffold struts with intraluminal scaffold dismantling. Therefore, the mechanism of late stent thrombosis/restenosis could be prevented by maximizing the scaffold dimension, embedding struts into plaque, and avoiding acute malapposition, which was strongly related to adverse event rates $[17,18]$.

Even though the importance of adherence to PSP is frequently emphasized, surprisingly, post-dilatation rates were about $<50 \%$, and varying PSP protocols were found in previously reported large-scale, randomized clinical trials and cohort studies [19]. In our study, extreme attention paid to the implantation technique by the operators was noted in both groups along with slightly lower post-dilatation rates in the eBVS group (pre-dilatation $100 \%$ in both groups, post-dilatation $99 \%$ in nBVS vs. $95 \%$ in eBVS). Also, despite both groups having similar lesion features, initially, more frequent use of scoring balloons in the eBVS group could be explained by the operator's concern for inadequate lesion preparation. 
A

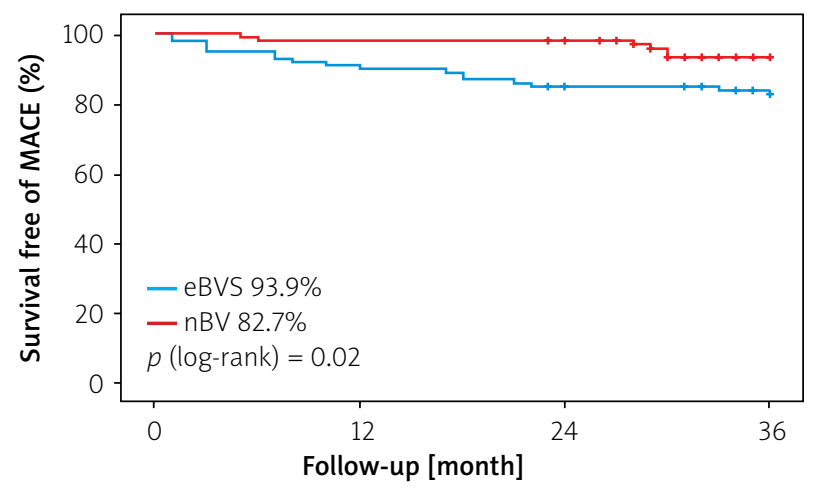

C

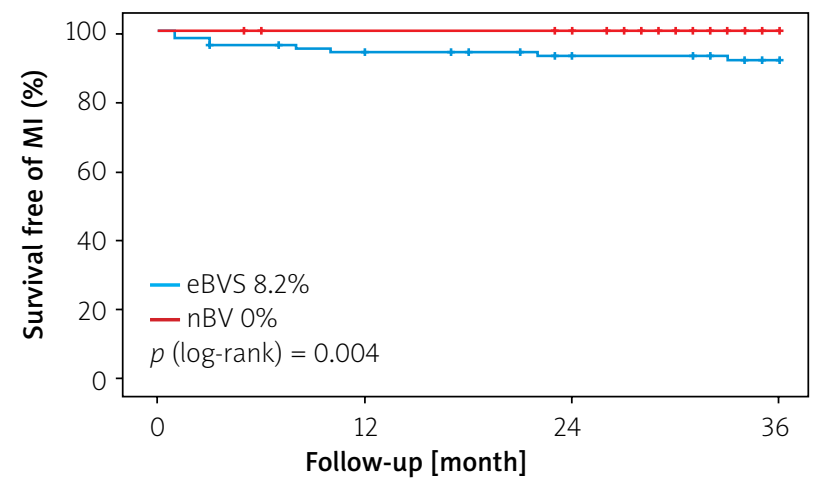

B

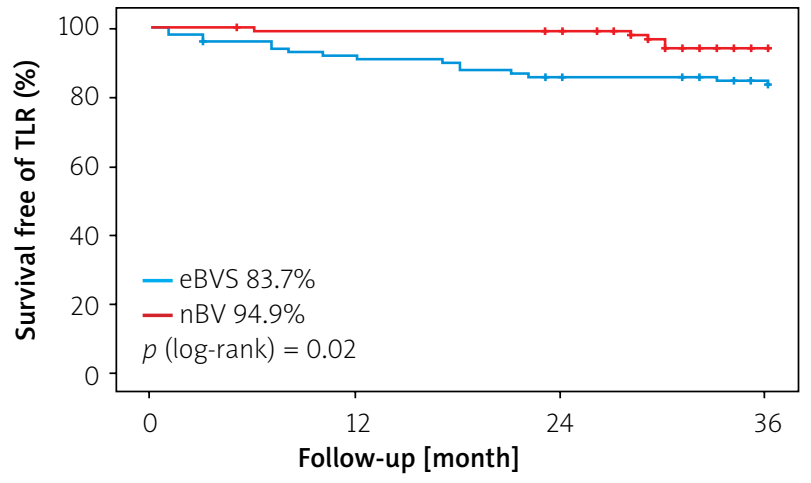

D

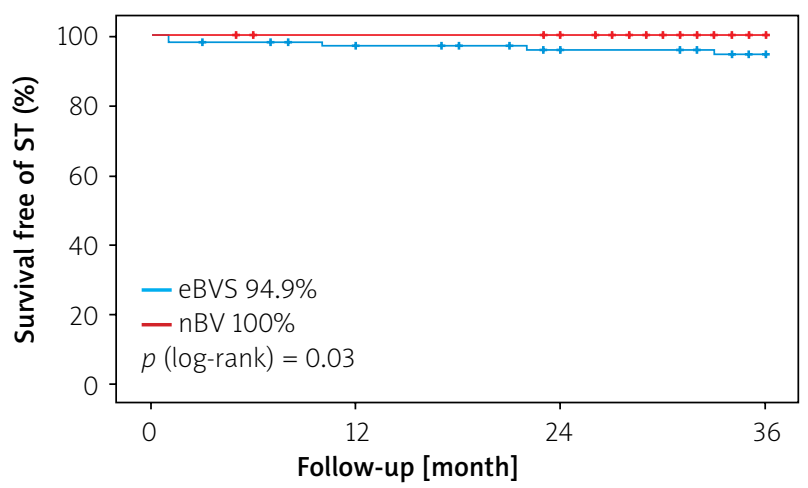

Figure 2. Kaplan-Meier survival curves at 3-years follow-up according to the eBVS and nBVS. A - MACE-free, B - TLR-free, C - MI-free, D - ST-free

MACE - major adverse cardiovascular events, TLR - target lesion revascularization, MI-myocardial infarction, ST-scaffold thrombosis, eBVS - everolimuseluting bioresorbable vascular scaffold, nBVS - novolimus-eluting bioresorbable vascular scaffold.

Naturally, most patients receiving eBVS were included in the early period of the study, while patients with nBVS were treated more recently. Thus, it could be speculated that the adoption of the optimal PSP protocol with growing experience over time might have also influenced the reported outcomes. Nevertheless, alarming rates of device thrombosis with eBVS from real life registries and randomized trials possibly have led to a trend towards more often utilization of nBVS lately.

Puricel et al. reported that in a multicenter registry of 1,305 patients implanted with eBVS, the rate of scaffold thrombosis declined significantly in patients when utilizing the strategy optimized for BVS rather than the DES-oriented implantation strategy for BVS procedures [8]. However, despite the optimal implantation practice, in the COMPARE-ABSORB trial, substantially increased risks of thrombotic events were found in complex de novo target lesions, defined as being at least $28 \mathrm{~mm}$ in length, in a small vessel, with a pre-existing total occlusion or a bifurcation [19]. Generally, optimization of the implantation technique is considered to be of paramount importance. We still detected higher MACE rates in the eBVS group despite PSP compliance. Therefore, the issue of whether or not to use the PSP protocol for better outcomes remains controversial.
Relatively greater elasticity of nBVS also contributes to lower stent fractures, together with its self-correction property providing better contact of struts and the vessel wall [20]. Routine aggressive postdilatation, unique structural features, shorter bioresorption time (95\% reduction in molecular weight by 1 year, with complete absorption by 2 years) of nBVS might be related to the absence of scaffold thrombosis noted in our study. A previous study from our center also showed no scaffold thrombosis with DESolve BVS [21].

In the randomized trials of the Absorb stent (Absorb Japan, Absorb China, Absorb III) more potent new generation P2Y12 inhibitors (ticagrelor, prasugrel) were used in a rate of $24 \%$ and $21 \%$ [22]. Approximately, more than half of the patients in both groups were given newer P2Y12 inhibitors, but early and late thrombotic events still occurred in the eBVS group beside very high PSP adherence.

The most relevant limitation of our study is the non-randomized nature, instead being a single center registry. Because of the small sample size, it is likely that it was not able to detect small differences between groups. So caution should be exercised in reaching definite conclusions. Lack of intravascular imaging guidance and systematic angiographic follow-up are other limita- 
tions of our study. Similarly, intravascular imaging was used in the minority of the patients in BVS trials [23]. Adequately powered randomized trials are needed to determine whether imaging guidance could reduce adverse events. No criteria were pre-defined to decide whether to implant either an nBVS or an eBVS, and thus selection bias cannot be ruled out. Both groups were treated in slightly different time periods, so the results might be biased by the operator experience and adoption of a better PSP implantation protocol. Nevertheless, our findings were obtained in a high-volume center, in which the operators paid meticulous attention to lesion preparation, BVS sizing, and post-dilatation. There was a difference in the loss at follow-up between groups, resulting in unequal duration of exposure to the risk of events. However, we sought to address this limitation by using Kaplan-Meier methods.

\section{Conclusions}

nBVS appears to be associated with good mid-term clinical outcomes compared to eBVS. More evidence is needed to evaluate long-term performance of novolimus-eluting biovascular platforms.

\section{Conflict of interest}

The authors declare no conflict of interest.

\section{References}

1. Serruys PW, Garcia-Garcia HM, Onuma Y. From metallic cages to transient bioresorbable scaffolds: change in paradigm of coronary revascularization in the upcoming decade? Eur Heart J 2012; 33: 16-25.

2. Caiazzo G, Kilic ID, Fabris E, et al. Absorb bioresorbable vascular scaffold: what have we learned after 5 years of clinical experience? Int J Cardiol 2015; 201: 129-36.

3. Ellis SG, Kereiakes DJ, Metzger DC, et al. Everolimus-eluting bioresorbable scaffolds for coronary artery disease. N Engl J Med 2015; 373: 1905-15.

4. Ali ZA, Gao R, Kimura T, et al. Three-year outcomes with the absorb bioresorbable scaffold: individual-patient-data meta-analysis from the ABSORB randomized trials. Circulation 2018; 137: 464-79.

5. Neumann FJ, Sousa-Uva M, Ahlsson A, et al. 2018 ESC/EACTS Guidelines on myocardial revascularization. Eur Heart J 2019; 40: 87-165.

6. Nef HM, Wiebe J, Foin N, et al. A new novolimus-eluting bioresorbable coronary scaffold: present status and future clinical perspectives. Int J Cardiol 2017; 227: 127-33.

7. Cutlip DE, Windecker S, Mehran R, et al. Clinical end points in coronary stent trials: a case for standardized definitions. Circulation 2007; 115: 2344-51.

8. Puricel S, Cuculi F, Weissner M, et al. Bioresorbable coronary scaffold thrombosis: multicenter comprehensive analysis of clinical presentation, mechanisms, and predictors. J Am Coll Cardiol 2016; 67: 921-31.

9. Ali ZA, Serruys PW, Kimura T, et al. 2-year outcomes with the Absorb bioresorbable scaffold for treatment of coronary artery disease: a systematic review and meta-analysis of seven randomised trials with an individual patient data substudy. Lancet 2017; 390: 760-72.

10. Abizaid A, Costa RA, Schofer J, et al. Serial Multimodality imaging and 2-Year clinical outcomes of the novel DESolve novolimus-eluting bioresorbable coronary scaffold system for the treatment of single de novo coronary lesions. JACC Cardiovasc Interv 2016; 9: 565-74.

11. Nef $\mathrm{H}$, Wiebe J. A multicenter post-marketing evaluation of the Elixir DESolve $\left({ }^{\circledR}\right)$ Novolimus-eluting bioresorbable coronary scaffold system: first results from the DESolve PMCF study. Catheter Cardiovasc Interv 2018; 92: 1021-7.

12. Mattesini A, Boeder N, Valente S, et al. Absorb vs. DESolve: an optical coherence tomography comparison of acute mechanical performances. Eurolntervention 2016; 12: e566-73.

13. Ormiston JA, Webber B, Ubod B, et al. An independent bench comparison of two bioresorbable drug-eluting coronary scaffolds (Absorb and DESolve) with a durable metallic drug-eluting stent (ML8/Xpedition). Eurolntervention 2015; 11: 60-7.

14. Barkholt TO, Webber B, Holm NR, et al. Mechanical properties of the drug-eluting bioresorbable magnesium scaffold compared with polymeric scaffolds and a permanent metallic drug-eluting stent. Catheter Cardiovasc Interv 2020; 96: E674-82.

15. Wiebe J, Dorr O, Ilstad H, et al. Everolimus- versus novolimus-eluting bioresorbable scaffolds for the treatment of coronary artery disease: a matched comparison. JACC Cardiovasc Interv 2017; 10: 477-85.

16. Ortega-Paz L, Capodanno D, Gori T, et al. Predilation, sizing and post-dilation scoring in patients undergoing everolimus-eluting bioresorbable scaffold implantation for prediction of cardiac adverse events: development and internal validation of the PSP score. Eurolntervention 2017; 12: 2110-7.

17. Stone GW, Abizaid A, Onuma Y, et al. Effect of technique on outcomes following bioresorbable vascular scaffold implantation: analysis from the ABSORB trials. J Am Coll Cardiol 2017; 70: 2863-74.

18. Gori T, Weissner M, Gonner S, et al. Characteristics, predictors, and mechanisms of thrombosis in coronary bioresorbable scaffolds: differences between early and late events. JACC Cardiovasc Interv 2017; 10: 2363-71.

19. Danzi GB, Bernelli C, Cerrato E. Outcomes of optimised implantation technique with bioresorbable scaffolds: a pooled analysis of ABSORB-IV and COMPARE-ABSORB trials. Cardiovasc Revasc Med 20120; 21: 559-61.

20. Schmidt W, Behrens P, Brandt-Wunderlich C, et al. In vitro performance investigation of bioresorbable scaffolds - standard tests for vascular stents and beyond. Cardiovasc Revasc Med 2016; 17: 375-83.

21. Cakal B, Cakal S, Karaca O, et al. Outcomes of the novolimus-eluting bioresorbable vascular scaffold in real world clinical practice. Minerva Cardioangiol 2020. DOI: 10.23736/S00264725.20.05138-5.

22. Bil J, Gil RJ. Bioresorbable vascular scaffolds-what does the future bring? J Thorac Dis 2016; 8: E741-5.

23. Serruys PW, Onuma Y. Dmax for sizing, PSP-1, PSP-2, PSP-3 or OCT guidance: interventionalist's jargon or indispensable implantation techniques for short- and long-term outcomes of Absorb BRS? Eurolntervention 2017; 12: 2047-56. 